\title{
Rayleigh beacon for measuring the surface profile of a radio telescope
}

\author{
S. Padin \\ California Institute of Technology, 1200 East California Boulevard, \\ Pasadena, California 91125, USA (spadin@ caltech.edu) \\ Received 16 September 2014; revised 31 October 2014; accepted 1 November 2014; \\ posted 4 November 2014 (Doc. ID 223243); published 25 November 2014
}

\begin{abstract}
Millimeter-wavelength Rayleigh scattering from water droplets in a cloud is proposed as a means of generating a bright beacon for measuring the surface profile of a radio telescope. A $\lambda=3 \mathrm{~mm}$ transmitter, with an output power of a few watts, illuminating a stratiform cloud, can generate a beacon with the same flux as Mars in $10 \mathrm{GHz}$ bandwidth, but the beacon has a narrow line width, so it is extremely bright. The key advantage of the beacon is that it can be used at any time, and positioned anywhere in the sky, as long as there are clouds. (C) 2014 Optical Society of America

OCIS codes: ～(290.5870) Scattering, Rayleigh; (090.2910) Holography, microwave; (120.6650) Surface measurements, figure.

http://dx.doi.org/10.1364/AO.53.008116
\end{abstract}

\section{Introduction}

The surface profile of a radio telescope is generally measured using holography. In conventional (coherent) holography, the amplitude and phase of the beam pattern are measured, and the aperture function is recovered by Fourier inversion [1,2]. The phase reference for the measurement is provided by an auxiliary antenna, which might be an interferometer element, or a small horn mounted on the main telescope [3-5]. Conventional holography is sensitive, so it is widely used for accurate surface measurements with high spatial resolution. The setting of most submillimeter-wavelength telescope surfaces is based on conventional holography. In out-of-focus (phase-retrieval) holography, the telescope power pattern is measured at different focus positions and the aperture function is recovered by modeling [6,7]. The spatial resolution tends to be low, because high signal-to-noise ratio is needed to recover the aperture function, but out-of-focus holography is useful for measuring low-order gravitational and thermal deformations [8].

$1559-128 \mathrm{X} / 14 / 348116-07 \$ 15.00 / 0$

(C) 2014 Optical Society of America
A bright source on a tower is often used for holography at a single, low elevation to support the initial setting of a telescope surface, but measurements over a wide elevation range are needed to fully optimize the telescope efficiency. Celestial sources are convenient for measuring the surface profile versus elevation [9]. Unfortunately, only the planets are bright enough for measurements of submillimeterwavelength telescopes, and there are long periods when no planets are visible. Transmitters on geostationary communications satellites can be used [10-12], but most operate below $20 \mathrm{GHz}$, where micrometer accuracy places unrealistic requirements on reflections and cross talk in the receiving system. Measurements that require high accuracy are usually made at millimeter wavelengths. Satellite beacons at $\sim 40 \mathrm{GHz}$ have been used to measure the IRAM $30 \mathrm{~m}$ and Heinrich Hertz Telescopes [3,12], but the satellites have failed or drifted out of orbit. A transmitter on a drone is another option, but stability of the transmitter position is a problem.

What is needed is a bright, stationary, millimeterwavelength beacon that can be positioned anywhere in the sky. There is as yet no radio equivalent of a sodium laser guide star, and millimeter-wavelength Rayleigh scattering from air molecules is too weak to 
be useful, but scattering from water droplets in clouds seems to be a practical option for measuring telescope surface errors in poor weather. This paper gives performance estimates for such a scheme. The paper begins with a description of scattering from air molecules and water droplets, followed by a calculation of the sensitivity of surface profile measurements using a Rayleigh beacon, and a discussion of the effects of beacon geometry. The paper ends with some comments on the practical implementation of a beacon.

\section{Rayleigh Scattering}

The scattering cross section of a single dielectric sphere that is much smaller than a wavelength is $[\underline{13}, \underline{14}]$

$$
\alpha=\frac{8 \pi}{3} a^{6} k^{4}\left(\frac{\epsilon-1}{\epsilon+2}\right)^{2},
$$

where $a$ is the radius of the sphere, $k=2 \pi / \lambda$, and $\epsilon$ is the dielectric constant of the sphere. In terms of the molecular polarizability $\gamma$, Eq. (1 $)$ becomes [15]

$$
\alpha=\frac{8 \pi}{3} \gamma^{2} k^{4} .
$$

If power $P$ is incident on a region containing dielectric spheres, the scattered flux is

$$
F \approx \frac{P \alpha N t}{2 \pi R^{2}},
$$

(e.g., in $\mathrm{Wm}^{-2}$ ) where $N$ is the number density of spheres, $R$ is the distance to the illuminated region, $t$ is the thickness of the region, and the scattered signal is assumed to be uniformly spread over $2 \pi$ str. The scattered signal is the sum of contributions from many randomly positioned spheres, so the signal is incoherent, much like thermal radiation from the disc of a planet.

The choice of wavelength for a Rayleigh beacon involves a compromise between transmitter power, which is higher at longer wavelengths, scattered power, which scales with $k^{4}$, and the cost of receiver components, which is higher at shorter wavelengths. Commercially available, high-power, pulsed klystrons can provide a few kilowatts peak output power at $\lambda=3 \mathrm{~mm}$, and a few tens of watts at $\lambda=1.5 \mathrm{~mm}$ [16]. The power scales much faster than $k^{-4}$, so a $\lambda=3 \mathrm{~mm}$ source is a better choice. Receivers are also much less expensive at $\lambda=3 \mathrm{~mm}$ because passive imaging systems operate in that band. For solid-state sources, which can produce a few hundred milliwatts at $\lambda=3 \mathrm{~mm}$, the power scales roughly as $k^{-4}$ [17], so the low cost of receiver components determines the beacon wavelength. All the calculations in this paper are for a $\lambda=3 \mathrm{~mm}$ beacon.

Scattering from air molecules is used to generate beacons for optical and infrared adaptive optics [18], but the $k^{4}$ term in Eqs. (1) and (2) leads to very small scattering at millimeter wavelengths. Oxygen and nitrogen molecules have $\gamma \sim 1 \AA^{3}$ [19], which gives a cross section of $1.6 \times 10^{-46} \mathrm{~m}^{2}$ at $\bar{\lambda}=3 \mathrm{~mm}$, and the number density of air molecules in the lower atmosphere is $N \approx 2 \times 10^{25} \mathrm{~m}^{-3}$, so scattering returns $3 \times 10^{-21}$ of the incident power per meter length of atmosphere. The scattering region can be made several kilometers long to increase the brightness of the beacon. A $1 \mathrm{~kW}, \lambda=3 \mathrm{~mm}$ source, illuminating a $5 \mathrm{~km}$ thick region at a distance of $10 \mathrm{~km}$, gives a scattered flux of $2.6 \times 10^{-23} \mathrm{Wm}^{-2}$. For comparison, Mars at apogee is a $200 \mathrm{~K}$ disc, 4 arcsec in diameter, with a flux density of $18 \mathrm{Jy}$ at $\lambda=3 \mathrm{~mm}$, so the flux is $9.0 \times 10^{-16} \mathrm{Wm}^{-2}$ in one polarization in a typical radio astronomy receiver bandwidth of $10 \mathrm{GHz}$. Rayleigh scattering from air molecules at $\lambda=3 \mathrm{~mm}$ is clearly too weak to be useful, even with a very high transmitter power.

Water droplets in clouds offer a source of larger scattering spheres. Stratiform clouds at altitudes below $\sim 6 \mathrm{~km}$ contain liquid water droplets with radius in the range $1-10 \mu \mathrm{m}$, and number density up to a few hundred per $\mathrm{cm}^{3}[20-23]$. At $\lambda=3 \mathrm{~mm}$, the dielectric constant of water is $\sim 6$ [24], so droplets with $a=$ $5 \mu \mathrm{m}$ have a cross section of $9.8 \times 10^{-19} \mathrm{~m}^{2}$. With $N=100 \mathrm{~cm}^{-3}$, scattering returns $9.8 \times 10^{-11}$ of the incident power, which is 10 orders of magnitude larger than for air molecules. To achieve the same flux as Mars in $10 \mathrm{GHz}$ bandwidth requires a $5 \mathrm{~W}$, $\lambda=3 \mathrm{~mm}$ source, illuminating a $100 \mathrm{~m}$ thick patch of cloud $3 \mathrm{~km}$ away. The power requirements are modest, so a beacon is practical when there are low-level clouds in the sky.

At altitudes above $\sim 6 \mathrm{~km}$, clouds contain ice crystals with 1000 to 10,000 times lower number density than the droplets in low-level clouds, but five to 10 times larger diameter [25]. The dielectric constant of ice at $\lambda=3 \mathrm{~mm}$ is about half that of liquid water [24]. For the same transmitter power, the scattered flux from a high-level cloud might be an order of magnitude smaller than from a low-level cloud, but the beacon is still bright enough to be useful.

The requirement for clouds is a disadvantage because telescopes for short radio wavelengths are usually on sites with little cloud, so a bright beacon is possible only in poor weather. Such a beacon is useful for occasional measurements to set the telescope surface, and to build models of thermal and gravitational deformation. Unfortunately, the beacon cannot be used for active control of the telescope surface during astronomical observations in the best weather. Relegating surface profile measurements to poor weather does have an advantage in that astronomy and surface measurements no longer have to share good weather.

\section{Sensitivity}

To calculate the sensitivity of a surface profile measurement, consider a telescope equipped with a two-horn interferometer as in Fig. 1 [26,27]. A beamsplitter ahead of the telescope focus provides 

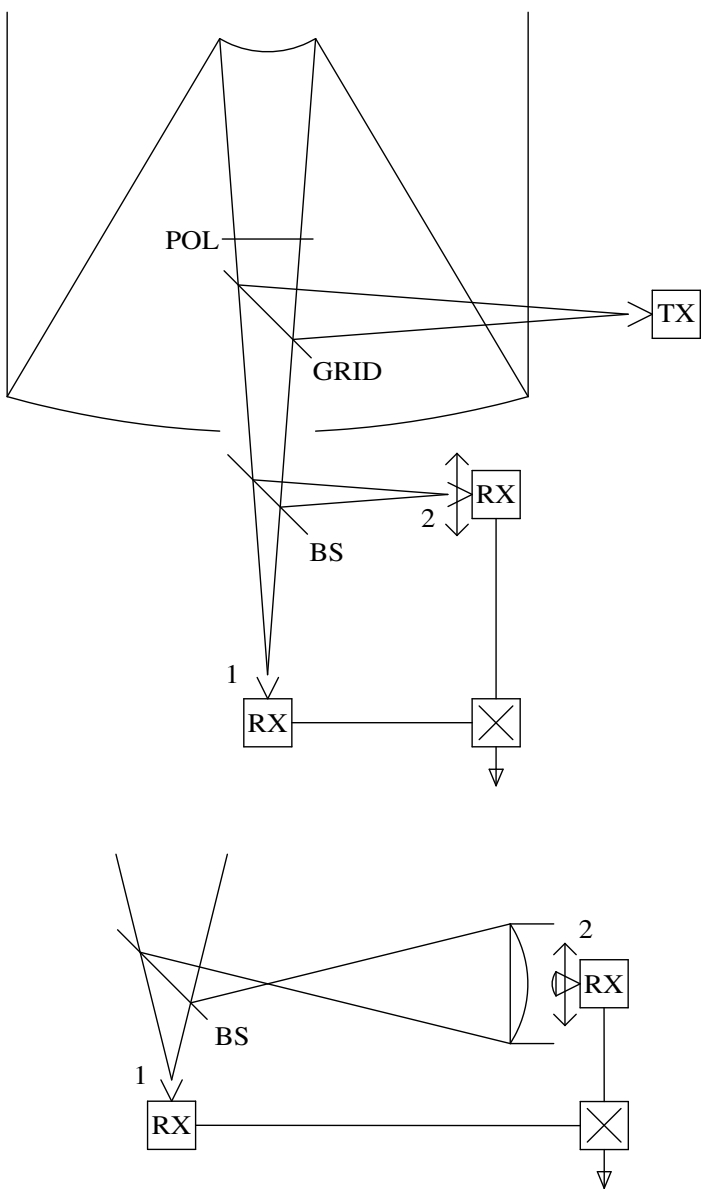

Fig. 1. Single-dish telescope with a beacon transmitter and a twohorn interferometer for measuring the amplitude and phase over the image (top) and interferometer details for measuring the amplitude and phase over the pupil (bottom). TX is the beacon transmitter, GRID is a wire grid that reflects the linearly polarized output from the transmitter, POL is a quarter-wave plate, BS is a beamsplitter, $\mathrm{RX}$ is a receiver, and $\mathrm{x}$ is a correlator.

two images of the source. Interferometer horn one captures the entire core of the first image, to provide a phase reference, while horn two is moved around the second image to measure the electric field. The measurement is equivalent to conventional holography with two identical telescopes, but here the two telescopes are generated by splitting the signal from a single dish. A similar scheme, but with a Fourier transform spectrometer and an incoherent detector, is used to measure the surface of the Caltech Submillimeter Observatory [28].

The field in the image is

$$
\mathbf{E}(\boldsymbol{\theta})=\mathbf{G}(\boldsymbol{\theta}) * \mathbf{V}(\boldsymbol{\theta}),
$$

where $\boldsymbol{\theta}$ is a point in the image, $\mathbf{G}(\boldsymbol{\theta})$ is the telescope angular response, $\mathbf{V}(\boldsymbol{\theta})$ is the source amplitude distribution, and $*$ is the convolution operator. Equation (4) applies even if the source is incoherent because the phase reference for the field measurement in Fig. 1 is the signal from the entire source. Fourier inversion of $\mathbf{E}(\boldsymbol{\theta})$ gives

$$
\mathbf{e}(\mathbf{r})=\mathbf{g}(\mathbf{r}) \times \mathbf{v}(\mathbf{r})
$$

where $\mathbf{r}$ is a point in the aperture, and $\mathbf{E}(\boldsymbol{\theta})$ and $\mathbf{e}(\mathbf{r})$, etc., are Fourier transform pairs. If the source is in the far field, $\mathbf{g}(\mathbf{r})$ is the aperture function, and the overall surface error is $\arg (\mathrm{g}) /(2 k)$. For a point source, which is generally the case in conventional holography, $\mathbf{V}(\boldsymbol{\theta})$ is a delta function, so $\mathbf{e}(\mathbf{r})=\mathbf{g}(\mathbf{r})$. When the source is in the near field, the simple Fourier rransform in Eq. (5) must be modified to account for the finite distance to the source [4].

The sensitivity calculation for an image-plane measurement must account for the shape of the image, but we can avoid this complication by changing to the pupil plane, where the signal is roughly uniform. Figure 1 shows a lens after the second image, to generate a pupil that is sampled by horn two. In this case, horn two measures the aperture function directly.

The wavefront measurement error in the pupil is

$$
\sigma=\frac{1}{k \times s / n},
$$

where $s / n$ is the signal to noise ratio at the correlator output. The signal amplitude is

$$
s=\left(P_{s 1} P_{s 2}\right)^{1 / 2},
$$

where $P_{s 1}$ and $P_{s 2}$ are the signal powers at the interferometer horns, and the noise is

$$
n=\frac{P_{n}}{(B \tau)^{1 / 2}},
$$

where $P_{n}$ is the noise power at each horn, $B$ is the predetection bandwidth, and $\tau$ is the integration time. For receivers with noise temperature $T$, the noise power is

$$
P_{n}=k_{B} T B,
$$

where $k_{B}$ is Boltzmann's constant.

Horn one captures the entire signal from the first image, so

$$
P_{s 1}=\frac{1}{2} F A_{1},
$$

where $F$ is the source flux (e.g., in $\mathrm{Wm}^{-2}$ ), $A_{1}$ is the area of the telescope, and the factor $1 / 2$ is the loss due to the beamsplitter. Horn two samples a patch of area $A_{2}$ in the pupil, so

$$
P_{s 2}=\frac{1}{2} F A_{2},
$$

where the factor $1 / 2$ is again due to the beamsplitter. $A_{2}$ must be a factor of a few smaller than a primary mirror segment in order to provide enough spatial resolution to recover the piston, tip, and tilt errors for each segment. Combining Eqs. (6-11) gives 


$$
\sigma=\frac{1}{k} \frac{2 k_{B} T B}{F\left(A_{1} A_{2}\right)^{1 / 2}} \frac{1}{(B \tau)^{1 / 2}},
$$

so high sensitivity requires a small receiver bandwidth and a beacon that emits a narrow line.

If a continuum source is used, the flux is

$$
F=\frac{1}{2} S B,
$$

where $S$ is the source flux density (e.g., in $\mathrm{WHz}^{-1} \mathrm{~m}^{-2}$ ), and the factor $1 / 2$ is for a single polarization receiver. In this case, the wavefront measurement error is

$$
\sigma=\frac{1}{k} \frac{4 k_{B} T}{S\left(A_{1} A_{2}\right)^{1 / 2}} \frac{1}{(B \tau)^{1 / 2}},
$$

so the receiver bandwidth should be as large as possible.

For a measurement using a Rayleigh beacon, the transmitter is pulsed, and the distance and thickness of the scattering region are defined by a range gate in the receiver. The range gate controls the period during which the field in the image or pupil is measured. The receiver bandwidth must be larger than the beacon line width and large enough to avoid smearing the gated signal from the beacon. The line width for a Rayleigh beacon is limited by random bulk motions in the cloud. (Thermal motion of the water droplets is only $\sim 1 \mu \mathrm{m} \mathrm{s}^{-1}$.) Radar measurements of drizzle droplets show speeds of $\sim 1 \pm 1 \mathrm{~ms}^{-1}$ $[29,30]$, and smaller droplets are unlikely to have random motions faster than the wind speed. A typical wind speed of $10 \mathrm{~ms}^{-1}$ gives a Doppler shift of $3 \mathrm{kHz}$ at $\lambda=3 \mathrm{~mm}$. The receiver range gate selects scattering from a region of thickness $t$, so the pulse width at the receiver is $t / c$, and the receiver bandwidth must be at least $c / t$, where $c$ is the speed of light. For $t=100 \mathrm{~m}$, the bandwidth must be $3 \mathrm{MHz}$.

From Eqs. (3) and (12), a surface profile measurement with $1 \mathrm{~m}$ spatial resolution on a $30 \mathrm{~m}$ telescope $\left(A_{1}=\pi(30 / 2)^{2} \mathrm{~m}^{2}\right.$ and $\left.A_{2}=\pi(1 / 2)^{2} \mathrm{~m}^{2}\right)$, using a $\lambda=3 \mathrm{~mm}$ Rayleigh beacon with $a=5 \mu \mathrm{m}$ water droplets $\left(9.8 \times 10^{-19} \mathrm{~m}^{2}\right.$ scattering cross section), $N=100 \mathrm{~cm}^{-3}, P=5 \mathrm{~W}, R=3 \mathrm{~km}$, and $t=100 \mathrm{~m}$, and a receiving system with $T=1000 \mathrm{~K}$ and $B=3 \mathrm{MHz}$, gives $\sigma=11 \mu \mathrm{m} \mathrm{rms}$ in $\tau=0.01 \mathrm{~s}$ (1 s elapsed time with $1 \%$ transmitter duty cycle). From Eq. (14), a similar measurement on Mars at $\lambda=3 \mathrm{~mm}$, with $B=10 \mathrm{GHz}$, gives $\sigma=62 \mu \mathrm{m} \mathrm{rms}$ in $\tau=1 \mathrm{~s}$, so the beacon measurement is 30 times faster.

\section{Beacon Geometry}

\section{A. Size}

If the beacon is larger than the telescope beam, the image is smeared, and the Fourier inverse of the image-plane field is tapered. The taper results in lower sensitivity to surface profile errors toward the edge of the aperture. Focus errors have a similar effect, so the telescope must be refocused for a beacon in the near field. The loss of sensitivity at the edge of the aperture is a problem for both the image-plane and pupil-plane measurements in Fig. 1.

A Rayleigh beacon that is launched by the telescope being measured has the same size and shape as the telescope beam, giving $\mathbf{V}(\boldsymbol{\theta})=|\mathbf{G}(\boldsymbol{\theta})|$ in Eq. (4). $\mathbf{V}(\boldsymbol{\theta})$ is real because the beacon is incoherent. At the telescope aperture, $\mathbf{v}(\mathbf{r})$ describes the coherence of the signal, but the intensity is uniform because the signal is the superposition of contributions from many incoherent point sources in the beacon. If the telescope is perfect, $\mathbf{g}(\mathbf{r})$ is a top hat function, and if the beacon is also in the far field, $\mathbf{G}(\boldsymbol{\theta})$ is real. In this case, $\mathbf{v}(\mathbf{r})=\mathbf{g}(\mathbf{r})$, so $\mathbf{v}(\mathbf{r})$ is a top hat function, the size of the aperture, and the aperture function is recovered with no taper.

A $30 \mathrm{~m}$ telescope that has been refocused at $R=3 \mathrm{~km}$ generates a $\lambda=3 \mathrm{~mm}$ beacon $\sim 20 \%$ larger than the far-field beamwidth of the telescope. In this case, the beacon is only $\sim 0.5 \mathrm{~m}$ in diameter, so the telescope is in the far field of the beacon and $\mathbf{v}(\mathbf{r}) \sim 2\left[J_{1}(p)\right] / p[31]$, where $p=\pi r b /(R \lambda), b$ is the beacon diameter, and $r=|\mathbf{r}|$. If $b=1.2 R \theta_{1}$, where $\theta_{1} \approx 1.22 \lambda / D_{1}$ is the far-field beamwidth, and $D_{1}$ is the telescope aperture diameter, and the sensitivity degradation at the edge of the aperture is $\mathbf{v}\left(D_{1} / 2\right) \sim 1 / 2$.

Loss of sensitivity toward the edge of the aperture can be avoided by reconstructing the wavefront error from wavefront tilt measurements that sample small patches in the pupil [32]. Laser guide star systems use this approach, but it is not easy to implement at millimeter wavelengths because the tilt measurements require a large-format detector array.

\section{B. Position}

The position of a Rayleigh beacon on the sky is defined by the launch telescope beam, while the distance and thickness of the beacon are defined by the receiver range gate. If the telescope being measured is also used to launch the beacon, and the droplet density is uniform, telescope pointing errors do not decenter the beacon on the receiver because the beacon moves with the telescope beam. Wavefront tip or tilt through the atmosphere is also compensated because the transmit and receive paths are the same. Unfortunately, real clouds have spatial variations in droplet size and number density, so the beacon moves as clouds drift through the telescope beam. If the wavefront is measured one point at a time, motion of the beacon adds noise to the measurement.

A displacement of the beacon on the sky tilts the wavefront, while a change in the distance to the beacon causes a defocus. The rms wavefront error due to these effects is 


$$
\begin{gathered}
\sigma_{\theta}=\frac{1}{4} \lambda \frac{\delta \theta}{\theta_{1}}, \\
\sigma_{R}=\frac{1}{16 \sqrt{3}} \frac{D_{1}^{2}}{R} \frac{\delta R}{R},
\end{gathered}
$$

where $\delta \theta$ is the angular displacement of the beacon, and $\delta R$ is the change in distance to the beacon. For $<10 \mu \mathrm{m} \mathrm{rms}$ wavefront error with $D_{1}=30 \mathrm{~m}$ and a $\lambda=3 \mathrm{~mm}$ beacon at $R=3 \mathrm{~km}$, the position of the beacon must be known within the beamwidth/75 and distance/1000. The receiver range gate limits motion of the beacon along the line of sight, but the range gate cannot be made very small because a thinner scattering region returns less power, and shorter beacon pulses require a larger receiver bandwidth.

The position of the beacon can be measured in the pupil plane using an array receiver to capture snapshots of the field over the pupil. A snapshot contains tip, tilt, and focus errors corresponding to the average position error of the beacon during the snapshot. Beacon position errors are degenerate with telescope pointing and focus errors, which can be measured easily using a celestial source, so the tip, tilt, and focus terms can be removed from the recovered aperture function, leaving only the higher-order terms that are useful for setting the telescope surface. The pointing and focus terms can be measured later, during normal astronomical observations in good weather, and corrected by adjusting the mount pointing and the position of the telescope secondary mirror.

It is not necessary to measure the entire pupil with a fully filled array receiver. A small, sparse array can be reconfigured between successive snapshots to build up full coverage of the pupil. The tip, tilt, and focus errors will vary from one snapshot to the next as the beacon moves around, so each snapshot must contain enough points to allow removal of the tip, tilt, and focus terms.

The error in the telescope surface measurement is determined by the error in each snapshot of the pupil, and the error in a single snapshot is limited by fitting errors for tip, tilt, and focus. The fitting error is in turn limited by surface profile errors, so the minimum error in the surface measurement is limited to a fraction of the actual surface error. The fitting error in a single snapshot is roughly $e / m^{1 / 2}$, where $e$ is the $\mathrm{rms}$ surface error, and $m$ is the number of elements in the array receiver, so a 10-element array is needed to keep the measurement error below $\sim 30 \%$ of the surface error.

\section{Shape}

A Rayleigh beacon is a column through the atmosphere, so it appears elongated when viewed off axis [33]. For a beacon that is launched by the telescope being measured, an observer in the telescope aperture sees the beacon as a line pointing away from the field center. The angular extent of the line is

$$
\delta \theta_{E} \approx \frac{r t}{R^{2}}
$$

For a $30 \mathrm{~m}$ telescope launching a beacon with $t=100 \mathrm{~m}$ at $R=3 \mathrm{~km}$, the apparent length of the beacon viewed from the edge of the telescope aperture is $R \delta \theta_{E}=0.5 \mathrm{~m}$, which is similar to the beacon diameter calculated in Section 4.A. Elongation displaces the center of the beacon, resulting in wavefront tilt of $\delta \theta_{E} / 2$. Integrating the tilt over $r$ gives the wavefront error

$$
\sigma_{E} \approx \frac{r^{2} t}{4 R^{2}}
$$

which is a defocus, so it can be removed from the recovered aperture function.

\section{Practical Considerations}

\section{A. Focus}

The focus error is removed in a surface measurement with a Rayleigh beacon, so the telescope does not need to be focused accurately, but it must be focused well enough to avoid smearing of the image and the nuisance of phase wraps across the pupil. Focusing is most easily accomplished by moving the secondary, so the secondary actuator must have enough range to accommodate a source just a few kilometers away.

\section{B. Calibration}

In a pupil-plane measurement, the recovered aperture function is contaminated by wavefront errors in the optical relay that generates the pupil. Gain and phase errors in the receiver have a similar effect, but can be more troublesome because the errors change with temperature and with motion of signal and local oscillator cables when the receiver is reconfigured. All of these errors can be calibrated by inserting a bright point source at the telescope focus just before the pupil.

\section{Transmitter Isolation}

If the beacon is launched by the telescope that is being measured, the receiver must be isolated from the transmitter to prevent damage to the receiver electronics. The receiver and transmitter must also be coupled to the telescope with low loss. It is unusual to have a high-power transmitter close to the low-noise receiver on a radio telescope, but this problem is common in radar and deep-space communication systems, and practical solutions do exist. The power at the receiver input must be kept below $\sim 1 \mathrm{~mW}$, so $\sim 40 \mathrm{~dB}$ isolation is needed for a $5 \mathrm{~W}$ transmitter. A polarization duplexer can provide the required isolation with only $\sim 0.1 \mathrm{~dB}$ loss [34]. In the scheme of Fig. 1 , if the polarization is linear vertical at the transmitter output, and left circular 
after the polarizer, the received signal is right circular, and the polarizer converts this to linear horizontal, which the grid directs to the receiver [35-37]. Transmitted power reflecting off the center of the secondary can illuminate the receiver directly, so the secondary may need a scattering cone, or hole, conjugate to the hole in the primary.

\section{Conclusions}

Rayleigh scattering from water droplets in a cloud can provide a bright, millimeter-wavelength beacon for measuring the surface profile of a telescope. The beacon can be positioned anywhere in the sky, so it is useful for measuring surface profile versus elevation, it can be launched by the telescope being measured, so pointing and tracking errors are removed, and it is extremely bright, so measurements can be made quickly. A $\lambda=3 \mathrm{~mm}$ transmitter with $5 \mathrm{~W}$ peak power and $1 \%$ duty cycle, illuminating a cloud with $\sim 10010$ - $\mu \mathrm{m}$-diameter droplets per $\mathrm{cm}^{3}$, allows a surface measurement of a $30 \mathrm{~m}$ telescope, with an accuracy of a few micrometers and $1 \mathrm{~m}$ spatial resolution, in an elapsed time of $10 \mathrm{~s}$ per point in the aperture. A sparse array receiver, with at least 10 elements, is needed to measure motion of the beacon due to variations in droplet size and number density, so a $30 \times 30$ surface profile map takes $\sim 15 \mathrm{~min}$. The array receiver adds some complexity to the system, but it is practical because of the availability of inexpensive, $\lambda=3 \mathrm{~mm}$, chip components for passive imaging.

Thanks to Jaap Baars, Richard Dekany, Richard Hills, Jeff Mangum, Ross Williamson, and David Woody for useful comments.

\section{References}

1. J. C. Bennett, A. P. Anderson, P. A. McInnes, and A. J. T. Whitaker, "Microwave holographic metrology of large reflector antennas," IEEE Trans. Antennas Propag. 24, 295-303 (1976).

2. P. F. Scott and M. Ryle, "A rapid method for measuring the figure of a radio telescope reflector," Mon. Not. R. Astron. Soc. 178, 539-545 (1977).

3. D. Morris, M. Bremer, G. Butin, M. Carter, A. Greve, J. W. Lamb, B. Lazareff, J. Lopez-Perez, F. Mattiocco, J. Penalver, and C. Thum, "Surface adjustment of the IRAM $30 \mathrm{~m}$ radio telescope," IET Microw. Antennas Propag. 3, 99-108 (2009).

4. J. W. M. Baars, R. Lucas, J. G. Mangum, and J. A. Lopez-Perez, "Near-field radio holography of large reflector antennas," IEEE Antennas Propag. Mag. 49(5), 24-41 (2007).

5. J. G. Mangum, J. W. M. Baars, A. Greve, R. Lucas, R. C. Snel, P. Wallace, and M. Holdaway, "Evaluation of the ALMA prototype antennas," Publ. Astron. Soc. Pac. 118, 1257-1301 (2006).

6. D. Morris, "Phase retrieval in the radio holography of reflector antennas and radio telescopes," IEEE Trans. Antennas Propag. 33, 749-755 (1985).

7. B. Nikolic, R. E. Hills, and J. S. Richer, "Measurement of antenna surfaces from in- and out-of-focus beam maps using astronomical sources," Astron. Astrophys. 465, 679-683 (2007).

8. B. Nikolic, R. M. Prestage, D. S. Balser, C. J. Chandler, and R. E. Hills, "Out-of-focus holography at the Green Bank Telescope," Astron. Astrophys. 465, 685-693 (2007).

9. D. Morris, J. W. M. Baars, H. Hein, H. Steppe, C. Thum, and R. Wohlleben, "Radio-holographic reflector measurement of the 30-m millimeter radio telescope at $22 \mathrm{GHz}$ with a cosmic signal source," Astron. Astrophys. 203, 399-406 (1988).

10. M. P. Godwin, E. P. Schoessow, and B. H. Grahl, "Improvement of the Effelsberg 100 meter telescope based on holographic reflector surface measurement," Astron. Astrophys. 167, 390-394 (1986).

11. D. J. Rochblatt and B. L. Seidel, "Microwave antenna holography," IEEE Trans. Microw. Theory Tech. 40, 1294-1300 (1992).

12. J. W. M. Baars, R. N. Martin, J. G. Mangum, J. P. McMullin, and W. L. Peters, "The Heinrich Hertz Telescope and the Submillimeter Telescope Observatory," Publ. Astron. Soc. Pac. 111, 627-646 (1999).

13. Lord Rayleigh, "On the transmission of light through an atmosphere containing small particles in suspension, and on the origin of the blue of the sky," Philos. Mag. 47(287), 375-384 (1899).

14. J. D. Jackson, Classical Electrodynamics, 2nd ed. (Wiley, 1975), Eq. (9.89).

15. J. D. Jackson, Classical Electrodynamics, 2nd ed. (Wiley, 1975), Eq. (4.70)

16. Communications \& Power Industries Canada Inc., www.cpii .com.

17. N. Priestley and N. Farrington, "Millimetre-wave Gunn diode technology and applications," UK Automated RF \& Microwave Measurement Society Conf., Oxfordshire, April 19-20, 2010.

18. C. A. Primmerman, D. V. Murphy, D. A. Page, B. G. Zollars, and H. T. Barclay, "Compensation of atmospheric optical distortion using a synthetic beacon," Nature 353, 141-143 (1991).

19. "NIST computational chemistry comparison and benchmark database," http://cccbdb.nist.gov.

20. H. R. Pruppacher and J. D. Klett, Microphysics of Clouds and Precipitation, 2nd ed. (Springer, 2010), Chap. 13.

21. N. L. Miles, J. Verlinde, and E. E. Clothiaux, "Cloud droplet size distribution in low-level stratiform clouds," J. Atmos. Sci. 57, 295-311 (2000)

22. G. M. Martin, D. W. Johnson, and A. Spice, "The measurement and parameterization of effective radius of droplets in warm stratocumulus clouds," J. Atmos. Sci. 51, 1823-1842 (1994).

23. H. Pawlowska, W. W. Grabowski, and J.-L. Brenguier, "Observations of the width of cloud droplet spectra in stratocumulus," Geophys. Res. Lett. 33, L19810 (2006).

24. V. G. Artemov and A. A. Volkov, "Water and ice dielectric spectra scaling at $0^{\circ} \mathrm{C}$," arXiv preprint arXiv:1308.1229 (2013).

25. M. Hess, P. Koepke, and I. Schult, "Optical properties of aerosols and clouds: the software package OPAC," Bull. Am. Meteorol. Soc. 79, 831-844 (1998).

26. S. von Hoerner, "Telescope surface measurement with two feeds," IEEE Trans. Antennas Propag. 26, 857-860 (1978).

27. D. B. Shenton and R. E. D. Hills, "A proposal for a U.K. mm wavelength astronomy facility," Sci. Res. Council, Appleton Lab., Nov. 1976.

28. E. Serabyn, T. G. Phillips, and C. R. Masson, "Surface figure measurements of radio telescopes with a shearing interferometer," Appl. Opt. 30, 1227-1241 (1991).

29. E. J. O'Connor, R. J. Hogan, and A. J. Illingworth, "Retrieving stratocumulus drizzle parameters using Doppler radar and lidar," J. Appl. Meteorol. 44, 14-27 (2005).

30. P. Kollias, W. Szyrmer, J. Remillard, and E. Luke, "Cloud radar Doppler spectra in drizzling stratiform clouds: 2. Observations and microphysical modeling of drizzle evolution," J. Geophys. Res. 116, D13203 (2011).

31. M. Born and E. Wolf, Principles of Optics, 6th ed. (Cambridge, 1998), Section 8.5.2.

32. W. H. Southwell, "Wave-front estimation from wave-front slope measurements," J. Opt. Soc. Am. 70, 998-1006 (1980).

33. J. W. Hardy, Adaptive Optics for Astronomical Telescopes (Oxford, 1998), Chap. 7.

34. J. L. Hirshfield, P. D. Kolchin, S. V. Kuzikov, and M. I. Petelin, "Quasi-optical antenna duplexer," in 25th Int. Conf. Infrared 
Millimeter Waves, Beijing, China, September 12-15, 2000, Conference Digest, pp. 405-406.

35. R. G. Fellers, "A circular-polarization duplexer for millimeter waves," Trans. Am. Inst. Electr. Eng. 78, 934937 (1960).

36. R. W. McMillan, C. W. Trussell, R. A. Bohlander, J. C. Butterworth, and R. E. Forsythe, "An experimental
$225 \mathrm{GHz}$ pulsed coherent radar," IEEE Trans. Microw. Theory Tech. 39, 555-562 (1991).

37. K. B. Cooper, N. Llombart, G. Chattopadhyay, B. Dengler, R. E. Cofield, C. Lee, S. Filchenkov, and E. Koposova, "A gratingbased circular polarization duplexer for submillimeter-wave transceivers," IEEE Microw. Wireless Compon. Lett. 22, 108-110 (2012). 